Research in Astron. Astrophys. 2010 Vol. 10 No. 12, 000-000

http://www.raa-journal.org http://www.iop.org/journals/raa

$\boldsymbol{R}$ esearch in

Astronomy and

Astrophysics

\title{
A Critique of Supernova Data Analysis in Cosmology
}

\author{
Ram Gopal Vishwakarma ${ }^{1}$ and Jayant V. Narlikar ${ }^{2}$ \\ 1 Unidad Académica de Matemáticas, Universidad Autónoma de Zacatecas, C.P. 98068, Zacatecas, \\ ZAC, Mexico; rvishwa@mate.reduaz.mx \\ 2 Inter-University Centre for Astronomy and Astrophysics, Pune 411007, India; jvn@ iucaa.ernet.in
}

Received [year] [month] [day]; accepted [year] [month] [day]

\begin{abstract}
Observational astronomy has shown significant growth over the last decade and has made important contributions to cosmology. A major paradigm shift in cosmology was brought about by observations of Type Ia supernovae. The notion that the universe is accelerating has led to several theoretical challenges. Unfortunately, although high quality supernovae data-sets are being produced, their statistical analysis leaves much to be desired. Instead of using the data to directly test the model, several studies seem to concentrate on assuming the model to be correct and limiting themselves to estimating model parameters and internal errors. As shown here, the important purpose of testing a cosmological theory is thereby vitiated.
\end{abstract}

Key words: cosmology: observations - supernovae Ia: general

Observations play a crucial role in all branches of science. Moreover, they are more important in cosmology where, on one hand, the events are non-repeatable and, on the other hand, the theoretical side is more speculative than laboratory physics, requiring guidelines from the observations by confronting them. The power of observations in cosmology is clear from the observations of supernovae ( $\mathrm{SNe}$ ) of type Ia, which dramatically changed, about a decade ago, the then standard picture of cosmology - of an expanding universe evolving under the rules of general relativity such that the expansion rate should slow down as cosmic time unfolds. Amazingly, the first generation of data showed that the rate of expansion of the universe is speeding up! This gave rise to a plethora of models to explain the agent driving the acceleration, as well as some possible modifications of general relativity. Theorists may debate the relative merits of various cosmic-acceleration theories: cosmological constant, dark energy, alternative gravity, anthropic arguments, etc., but it is ultimately up to the observations to decide which theory is correct.

However, we notice a recent unfortunate trend in the analysis of SNe Ia data, which departs from an objective assessment of a theory by observations. Everybody would agree that the first step in fitting the observational data to a theory is to check whether the theory is consistent with the data for viable values of its free parameters (if any). In order to do this, there are two standard ways: (i) the Bayesian approach, which gives a relative rather than an absolute measure of how good a theory is, and hence is more appropriate for comparison between competing theories; (ii) the maximum likelihood approach, which is more commonly used for hypothesis testing. Under this approach, one minimises $\chi^{2}$ which measures the deviations of the theoretical predictions from the observations. In the present case of the observations of magnitude $(m)$ and redshift $(z)$ of SNe Ia, the $\chi^{2}$ is given by the value

$$
\chi^{2}=\sum_{j=1}^{N} \frac{\left[m_{\mathrm{t}}\left(z_{j} ; \text { parameters }\right)-m_{\mathrm{o}, j}\right]^{2}}{\sigma_{m_{j}}^{2}},
$$


where $m_{\mathrm{t}}\left(z_{j}\right.$; parameters $)$ is the theoretical value of the magnitude at redshift $z_{j}$ of the $j$ th supernova predicted by a model which is given in terms of its parameters. The observed magnitude of the $j$-th SN is $m_{\mathrm{o}, j}$. The variance $\sigma_{m_{j}}^{2}$ represents the combined uncertainty in the observed magnitude of the $j$ th supernova arising from the uncertainties in the different variables, for example, lensing, dust extinction, peculiar velocity of the host galaxy, etc. By Taylor expanding $m$ around its mean value and by recalling that the variance of $(m) \equiv \sigma_{m}^{2}=\left\langle m^{2}\right\rangle-\langle m\rangle^{2}$, one can write the combined uncertainty of $m$ in terms of the uncertainties in its variables, say, $x_{i}$ :

$$
\sigma_{m}^{2}=\sum_{i}\left(\frac{\partial m}{\partial x_{i}}\right)^{2} \sigma_{x_{i}}^{2}+\sum_{i} \sum_{k \neq i}\left(\frac{\partial m}{\partial x_{i}}\right)\left(\frac{\partial m}{\partial x_{k}}\right) \operatorname{cov}\left(x_{i}, x_{k}\right),
$$

where $\operatorname{cov}\left(x_{i}, x_{k}\right)$ is the covariance between the variables $x_{i}$ and $x_{k}$, which vanishes for any uncorrelated variables. It is obvious from equation (1) that if the model satisfactorily represents the data, the difference between the predicted magnitude and the observed one at each data point should be roughly the same size as the measurement uncertainties and each data point would contribute roughly one to $\chi^{2}$, giving the sum that roughly equals the number of data points $N$ (more correctly $N$-number of fitted parameters $\equiv$ number of degrees of freedom 'DoF'). If $\chi^{2}$ is much larger, the fit is considered bad. A more quantitative assessment of the goodness-of-fit is given in terms of the $\chi^{2}$-probability. If the fitted model provides a typical value of $\chi^{2}$ as $x$ at $n$ DoF, this probability is given by

$$
P(x, n)=\frac{1}{\Gamma(n / 2)} \int_{x / 2}^{\infty} e^{-u} u^{n / 2-1} \mathrm{~d} u .
$$

Thus, $P(x, n)$ gives the probability that a model which does fit the data at $n$ DoF, would give a value of $\chi^{2}$ as large as $x$ or larger. Qualitatively, $P$ represents the probability of finding a worse fit to the data. If $P$ is very small, the fit is not acceptable. For example, if we get a $\chi^{2}=20$ at 4 DoF for some model, then the hypothesis that the model describes the data satisfactorily is unlikely, as the probability $P(20,4)=0.0005$ is very small. However, the $\chi^{2}$-probability $P$ holds strictly only when the measurement errors are normally distributed. In reality, they are not. In most cases, the effect of non-Gaussian errors is to create an abundance of outlier points, which decrease the probability $P$. It is due to this reason that usually models with $P>0.001$ are considered acceptable. One should proceed to estimate the parameters of the model only after examining if the model has a credible goodness-of-fit, in the absence of which, the estimated parameters of the model (and their estimated uncertainties) have no meaning at all.

Nevertheless, we noticed (see also Vishwakarma 2007) that recently an approach has been followed to analyze the SNe Ia data which does not respect the standard procedure described above. Initiated by Astier et al. (2006), this approach simply assumes (rather than examines) that the standard cosmology ( $23 \%$ of dark matter and $72 \%$ of dark energy) is consistent with the $\mathrm{SNe}$ Ia observations and limits itself to parameter estimation. Under this new approach, $\chi^{2}$ is calculated from

$$
\chi^{2}=\sum_{j=1}^{N}\left[\frac{\left\{m_{\mathrm{t}}\left(z_{j} ; \text { parameters }\right)-m_{\mathrm{o}, j}\right\}^{2}}{\sigma_{m_{j}}^{2}+\sigma_{\mathrm{int}}^{2}}\right]
$$

where $\sigma_{\text {int }}$ is the (unknown) intrinsic dispersion of the SN absolute magnitude (sometimes termed $\sigma_{\text {sys }}$, unknown systematic uncertainties) which is used as an adjustable free parameter in order to obtain

$\chi^{2} / \mathrm{DoF}=1$. Clearly this new approach does not test the model under consideration. Rather, it assumes that the model is correct and goes on to find the best value for intrinsic dispersion. In principle, any model applied to the data in this way will determine $\sigma_{\text {int }}$ and the purpose of testing the model or estimating its parameters is lost.

One may consider $\chi^{2} / \mathrm{DoF}=1$ to estimate $\sigma_{\text {int }}$ from the nearby SNe alone as $\sigma_{\text {int }}$ estimated from the nearby SNe alone and that estimated from the whole sample, do not differ significantly. For example, $\sigma_{\text {int }}=0.15 \pm 0.02$ estimated from the fit of the nearby sample $(z \leq 0.1)$ of Astier et al. (2006) 
appears to be statistically consistent with $\sigma_{\text {int }}=0.13 \pm 0.02$ estimated from their whole sample and $\sigma_{\text {int }}=0.12 \pm 0.02$ estimated from their distant SNe alone. However, one can always argue why a cosmological theory should be assumed to be correct (i.e., $\chi^{2} / \mathrm{DoF}=1$ ) even for the nearby SNe, even though all the Robertson-Walker models reduce to the $m_{\mathrm{t}}=5 \log z+$ constant for low $\mathrm{z}$. In addition, a more reasonable way to introduce $\sigma_{\text {int }}$ in the theory, is to use independent measurement uncertainties $\sigma_{\mathrm{int}, \mathrm{j}}$ from the individual $\mathrm{SNe}$ and use the correct expression for $\sigma_{m}^{2}$ given by equation (2) where $\sigma_{\mathrm{int}}$ enters through the term $\left(\partial m / \partial x_{\text {int }}\right)^{2} \sigma_{\text {int }}^{2}$.

Of course, one can estimate $\sigma_{\text {int }}$ (if one is just interested in that) from all (high- as well as lowredshift) SNe data by assuming that a particular theory (here the standard cosmology), already tested, must be consistent with the data (i.e., $\chi^{2} / \mathrm{DoF}=1$ ). This is fine if the theory is well established and is already tested by other independent ways. Then we are not interested in testing the already established theory, but rather we want to estimate, from(4), some parameters of the data (here $\sigma_{\text {int }}$ ) which we could not decipher from the observations. This procedure is followed in many branches of physics. But what are the other observations which predict dark energy independently and conclusively? To date, it is only the SNe Ia observations (if taken independently, i.e., not combined with other observations). The only other precise observations are the anisotropy measurements of the CMB made by the WMAP project. However, taken at their face values, the only relevant prediction of the WMAP observations is of a flat geometry, and the decelerating models (like the Einstein-de Sitter model: $\Omega_{m}=1, \Lambda=0$ ) also successfully explain them (see for example, Blanchard 2003; Blanchard et al. 2005; Vishwakarma 2003). Observations on gravitational lensing, quasars, galaxy clusters, gamma ray bursts, etc., are not precise enough. Age considerations depend heavily on the observations of $H_{0}$ and $\Omega_{m}$ which have wide-ranging degeneracy. Moreover, the best estimates of these parameters also include those which are estimated from the SNe Ia observations themselves. In fact, a conclusive prediction of dark energy only comes from the SNe Ia case. To make the situation worse, there have been claims (Wei 2010) of some conflicts between recent SNe Ia data sets Union (Kowalski et al. 2008) and Constitution (Hicken et al. 2009); these disagree not only with CMB and BAO (baryon acoustic oscillation) studies, but also with other SNe Ia data sets.

So, if we do not have conclusive independent evidence for dark energy, what is the meaning of $\sigma_{\text {int }}$ (and other parameters) estimated from this model, following this new approach to data analysis? The current concordance model in cosmology (also known as $\Lambda$ CDM cosmology) has 18 parameters (one can extract these parameters from Hinshaw et al. 2009 and Kowalski et al. 2008), 17 of which are independent. Thirteen of these parameters are well fitted to the observational data; the other four remain floating. This situation is very far from healthy, not only because of very few observational tests which support the standard cosmology, that it calls for more observational tests, but also because of the controversial theoretical aspects. The well known fine tuning and coincidence problems related to dark energy, as well as its extremely speculative character (particle physics even fails to identify a theory for dark energy), have invoked possible modifications of general relativity. Additionally on the observational side, there remains a lack of understanding of some issues related to SNe Ia observations causing a number of systematic uncertainties (which are likely to depend on redshift) that could affect the use of SNe Ia as standard candles in such cosmological probes. For example, one can mention the evolution of luminosity in SNe Ia (Dominguez et al. 1998; Hoflich et al. 1998; Drell et al. 2000; Timmes, Brown \& Truran 2003) and extinction of SNe light by dust which is still a poorly understood phenomenon (Holwerda 2008; Albrecht et al. 2006; Conley et al. 2007). Finally, it may be noted that different methods for analyzing SNe Ia data, in order to estimate magnitudes, do not seem entirely consistent with each other, causing systematic error concerns. For example, two different light-curve fitters, the multi-color light-curve shape (MLCS) method and the spectral-adaptive light-curve template (SALT) method, give significantly different estimates of magnitudes of the individual SNe; besides, the magnitudes estimated by the SALT fitter acquire a degree of cosmology-dependence (Frieman 2008). This situation warrants more rigorous tests of the theories as well as the observations. Certainly it suggests caution in regard to accepting the standard model as proven.

Initiated by the SuperNova Legacy Survey "SNLS" (Astier et al. 2006) and followed by Union1 (Kowalski et al. 2008), Union2 (Amanullah et al. 2010), Sloan Digital Sky Survey-II “SDSS-II” (Kessler 
et al. 2009) and SNLS 3-year (Guy et al. 2010) data sets, this flawed approach has already acquired the status of 'standard practice' in Lampeitl et al. (2010). The harmful side effect of the current approach is that even if the authors do not follow this approach, usually they do not care to check if their model fits the data or not, and limit themselves to estimating the parameters of the model either by only using the $\mathrm{SNe}$ Ia data or by combining the SNe Ia data with other observations. This is clear from the results of the Constitution data (Hicken et al. 2009), the largest sample so far consisting of 397 SNe Ia: although the authors do not follow the new approach, they do not seem to notice that the theory does not fit the data well! One can calculate from their Table 1 that the best-fitting $\Lambda$ CDM model, with $\Omega_{\mathrm{m}}=1-\Omega_{\Lambda}=$ 0.29 , gives $\chi^{2} / \mathrm{DoF}=465.5 / 395$ with a meager probability $P=0.83 \%$; and so the estimated model can be ruled out at more than a $99 \%$ confidence level! Other cosmologies also have a similar fit.

Finally, we emphasize that our arguments here offer a criticism of the statistical technique used for analyzing the data rather than a criticism of the standard model itself.

\section{References}

Albrecht A. et al., 2006, astro-ph/0609591.

Amanullah R., et al. 2010, ApJ. 716, 712.

Astier P., et al., 2006, A \& A, 447, 31.

Blanchard A., et al., 2003, A \& A, 412, 35.

Blanchard A., 2005, 'Cosmological Interpretation from High Redshift Clusters Observed Within the XMM-Newton $\Omega$-Project', Proceedings of DARK 2004, the Fifth International Heidelberg Conference, October 3-9, Texas A\&M University [preprint: astro-ph/0502220].

Conley A., Carlberg R. G., Guy J., Howell D. A., Jha S., Riess A. G., Sullivan M., 2007, ApJ 664, L13.

Dominguez I., et al, 'Type Ia Supernovae: Influence of the Progenitor on the Explosion', Proceedings of "Nuclei in the Cosmos V" Volos, Greece (1998) [preprint:astro-ph/9809292].

Drell P. S., Loredo T. J. and Wasserman I., 2000, ApJ, 530, 593.

Frieman, J. A. 2008, in AIP Conf. Ser. 1057, 87 (arXiv: 0904.1832).

Guy, J., et al. 2010, arXiv: 1010.4743 .

Hicken M., et al., 2009, ApJ, 700, 1097.

Hinshaw G., et al., 2009, ApJ. Suppl., 180, 225.

Hoflich P., Wheeler J. C. \& Thielemann F. K., 1998, ApJ, 495, 617.

Holwerda B. W., 2008, MNRAS, 386, 475.

Kessler R., et al., 2009 (to appear in ApJS), arXiv:0908.4274,

Kowalski M., et al., 2008, ApJ, 686, 749.

Lampeitl H., et al., 2009, (to appear in MNRAS), arXiv:0910.2193.

Timmes F. X., Brown E. F. \& Truran J. W., 2003, ApJ, 590, L83.

Vishwakarma R. G., 2003, MNRAS, 345, 545.

Vishwakarma R. G., 2007, Int. J. Mod. Phys. D, 16, 1641.

Wei H., 2010, Phys. Lett. B, 687, 286. 